\title{
Distant stable direct orbits around the Moon
}

\author{
O. C. Winter and E. Vieira Neto ${ }^{\star}$
}

\author{
Grupo de Dinâmica Orbital \& Planetologia, UNESP, C.P. 205, Guaratinguetá, CEP 12500-000, SP, Brazil \\ e-mail: ocwinter@feg.unesp.br; ernesto@feg.unesp.br
}

Received 5 December 2001 / Accepted 28 June 2002

\begin{abstract}
In the present work we explore regions of distant direct stable orbits around the Moon. First, the location and size of apparently stable regions are searched for numerically, adopting the approach of temporary capture time presented in Vieira Neto \& Winter (2001). The study is made in the framework of the planar, circular, restricted three-body problem, Earth-Moonparticle. Regions of the initial condition space whose trajectories are apparently stable are determined. The criterion adopted was that the trajectories do not escape from the Moon during an integration period of $10^{4}$ days. Using Poincaré surface of sections the reason for the existence of the two stable regions found is studied. The stability of such regions proved to be due to two families of simple periodic orbits, $h 1$ and $h 2$, and the associated quasi-periodic orbits that oscillate around them. The robustness of the stability of the larger region, $h 2$, is tested with the inclusion of the solar perturbation. The size of the region decreases, but it is still significant in size and can be useful in spacecraft missions.
\end{abstract}

Key words. planets and satellites: formation - celestial mechanics - astrometry

\section{Introduction}

Considering the framework of the restricted three-body problem, the existence of regions of distant stable retrograde orbits is well known (Broucke 1968; Hénon 1970; Jefferys 1971; Winter 2000; Winter \& Vieira Neto 2001). Such regions have been used in order to try to explain the origin of irregular planetary satellites (Huang \& Innanen 1983; Brunini 1996; Vieira Neto \& Winter 2001). In the case of the Earth-Moonparticle system (Winter 2000) these stable regions can conveniently be used in spacecraft missions to the Moon. However, in many cases those regions cannot be taken into account for missions with specifications that demand a direct trajectory. In those cases it is important to know the regions of distant stable direct orbits. Therefore, in the present work we address the problem of the location and size of the regions of distant stable direct orbits around the Moon.

The zero velocity curve associated with the Lagrangian point $L 1$ defines a physical region around the Moon (Fig. 1) known as the "Roche lobe". This region is usually accepted as the furthest limit of stable direct orbits around the Moon. Such a limit is also defined in terms of the value of the Jacobi constant at $L 1, C j(L 1)=3.2010$ for the Earth-Moon case. For trajectories with $C j>C j(L 1)$ it is not possible to occur escape from the region around the Moon or capture from the other regions. Our main goal is to find regions of stable trajectories with $C j<C j(L 1)$ and identify the reason for such stability.

Send offprint requests to: O. C. Winter,

e-mail: ocwinter@feg.unesp.br

* UNESP Post Doctor Program
In order to explore such regions we adopted the temporary capture time approach (Vieira Neto \& Winter 2001); the technique and the results are presented in the next section. In Sect. 3 we use the Poincaré surface of section technique to study the reason for the existence of the two stable regions found. Two families of periodic orbits are identified, $h 1$ and $h 2$. In Sect. 4, a diagram of stability in terms of Jacobi constant and position is generated (Winter 2000). In the last section, the robustness of the stability of the larger region, $h 2$, is tested with the inclusion of the solar perturbation.

\section{Stable region: Capture time analysis}

In a previous paper (Vieira Neto \& Winter 2001) we studied the problem of temporary gravitational capture of hypothetical satellites of Uranus. The approach adopted was to compute the capture times for a significant part of the initial condition space. One of the important features presented in the results was the regions of prisoner trajectories, trajectories that did not escape for an integration period of $10^{5}$ years. Winter \& Vieira Neto (2001) showed that the prisoner trajectories are actually stable trajectories. So, this approach proved to be an excellent way to detect regions of stability. In the following we describe the methodology of gravitational capture time.

The idea is based on the two-body orbital energy monitoring. The orbital energy is defined as:

$E=\frac{V^{2}}{2}-\frac{\mu}{r}$ 
where $V$ is the particle-Moon relative velocity in the fixed system, $r$ is the particle-Moon distance and $\mu$ is the mass parameter of the Moon.

Due to the perturbation of the third body (Earth), the twobody energy (Moon-particle) is not constant. By monitoring $E$, it is possible to have a clue of which primary has the dominant gravitational influence on the particle's trajectory. The changing of the energy's sign indicates that the osculating orbit changed from a closed orbit to an open one (escape) or from an open orbit to a closed one (capture). However, this effect is temporary and the trajectories that present a relatively large capture time (longer than the integration time) are strong candidates to be regular stable orbits.

The procedure is to explore the space of initial conditions and present the results in terms of diagrams indicating the region explored and the initial conditions that are labeled as prisoner trajectories. Each graphic is obtained with a grade of the semi-major axis and eccentricity, maintaining the values of the other orbital elements constant. The orbital elements are all relative to the Moon. The initial value of the semi-major axis, $a$, is chosen from $10000 \mathrm{~km}$ to $50000 \mathrm{~km}$, in steps of $100 \mathrm{~km}$. The eccentricity initial value, $e$, is chosen from 0 to 0.99 , in steps of 0.01 . So, each point in the graphic corresponds to one trajectory. Moreover, each trajectory is integrated with a negative time step from zero to -5000 days. Due to the configuration of the initial orbital elements, the initial two-body energy has a negative value (closed orbit). With the perturbation of the third body (the Earth), the energy changes its value. When the value of the orbital energy changes to a positive value (open orbit) the integration is interrupted and the time is stored. This time is called the capture time. Actually, the length of time for a temporary capture is higher than this value. The initial conditions satisfy the mirror theorem (Roy \& Ovenden 1955) for almost all the cases considered $(\omega=0$ or $\pi$, where $\omega$ is the argument of pericenter), which means that in those cases the precisely correct values for the capture time are twice the values of the integration time. The integration could also stop if the particle had a collision with the planet or the integration

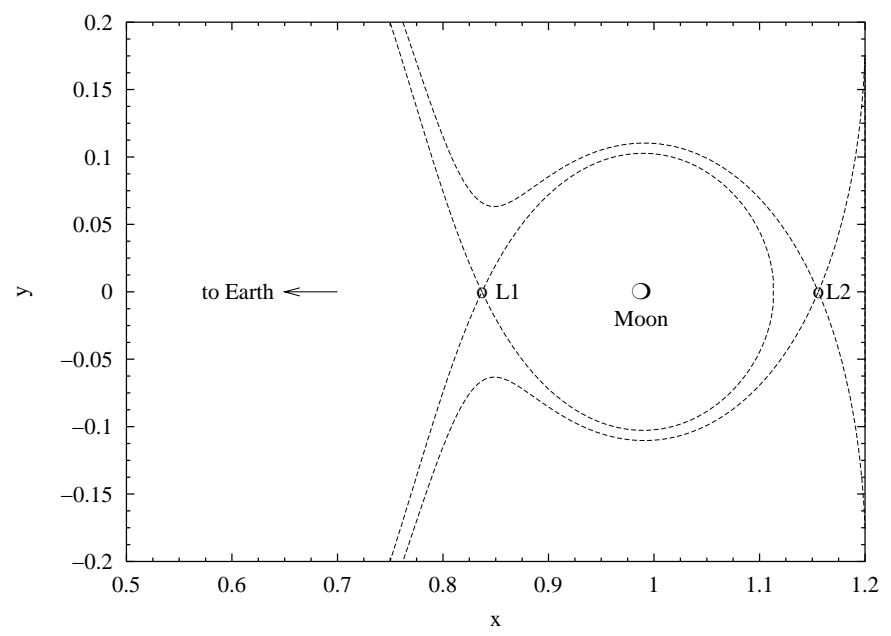

Fig. 1. Diagram indicating the region around the Moon and the Lagrangian points $L_{1}$ and $L_{2}$. The dashed lines correspond to the zero velocity curves that touch the points $L_{1}$ or $L_{2}$. time surpassed 5000 days. If the particle does not escape in the period of 5000 days its trajectory is called prisoner.

Figure 2 shows a diagram of the initial conditions in terms of $a$ versus $e$. The black lines in the figure stand for the zero velocity curves with $C j=C j(L 1)$ (most left line) and with $C j=C j(L 2)$ (two lines inside the gray region). The top half of this diagram $(e>0)$ corresponds to conditions at pericenter at opposition ( $\omega=0$ and $\tau=0$, where $\tau$ is the pericenter's epoch), while the other half ( $e<0$, this sign is just symbolic) corresponds to conditions at apocenter at opposition $(\omega=\pi$ and $\tau=P / 2$, where $P$ is the osculating orbital period). Initial conditions whose trajectories presented capture times longer than $10^{4}$ days are indicated by the white areas (inside the gray region) labeled $h 1$ and $h 2$.

We also explored regions of the initial condition space for prograde orbits at pericenter for a range of values of the argument of pericenter, $0<\omega<\pi$, with $\Delta \omega=\pi / 6$. No other region of prisoner trajectories was found for such values of $\omega$.

\section{Identification of the stable orbits}

The reason for the existence of the two apparently stable regions, $h 1$ and $h 2$, is now studied using the technique of Poincaré surfaces of section. In the following the method and the results are presented.

In order to determine the orbital elements of a particle at any instant it is necessary to know its position $(x, y)$ and velocity $(\dot{x}, \dot{y})$; these correspond to a point in a four dimensional phase space. The existence of the Jacobi constant implies the existence of a three dimensional surface in the four dimensional space. For a fixed value of the Jacobi constant only three of the four quantities are needed, say $x, y$ and $\dot{x}$, since the other one, $\dot{y}$ is determined, up to a change in sign, by the Jacobi constant. By defining a plane, say $y=0$, in the resulting three dimensional space, the values of $x$ and $\dot{x}$ can be plotted every time the particle has $y=0$. The ambiguity in the sign of $\dot{y}$ is removed by considering only those crossing with a fixed sign of $\dot{y}$. The section is obtained by fixing a plane in phase space and plotting the points when the trajectory intersects this plane in a particular direction. This technique is good at determining the regular or chaotic nature of the trajectory. In the Poincaré map, quasiperiodic orbits appear as closed well-defined curves. Periodic orbits appear as isolated single points inside such islands. Any "fuzzy" distribution of points in the surface of section implies that the trajectory is chaotic.

This is the method of the Poincaré surface of section or the Poincaré map. It has been largely used to determine the location and size of regular and chaotic regions in the phase space of the circular restricted three-body problem.

In order to explore the region of apparent stability shown in Fig. 2 we produced about thirty Poincaré surfaces of section. The range of $C_{j}$ considered coincide with the range needed to cover the white areas, $h 1$ and $h 2$, shown in Fig. 2 (3.1800 $\leq$ $\left.C_{j} \leq 3.2040\right)$. A sample of the Poincaré surfaces of section generated are given in Figs. 3 and 4. The size of the largest islands indicate the region of stability for each Jacobi constant. From the whole set of Poincaré surfaces of section considered we verified that the regions $h 1$ and $h 2$ (Fig. 2) are actually 


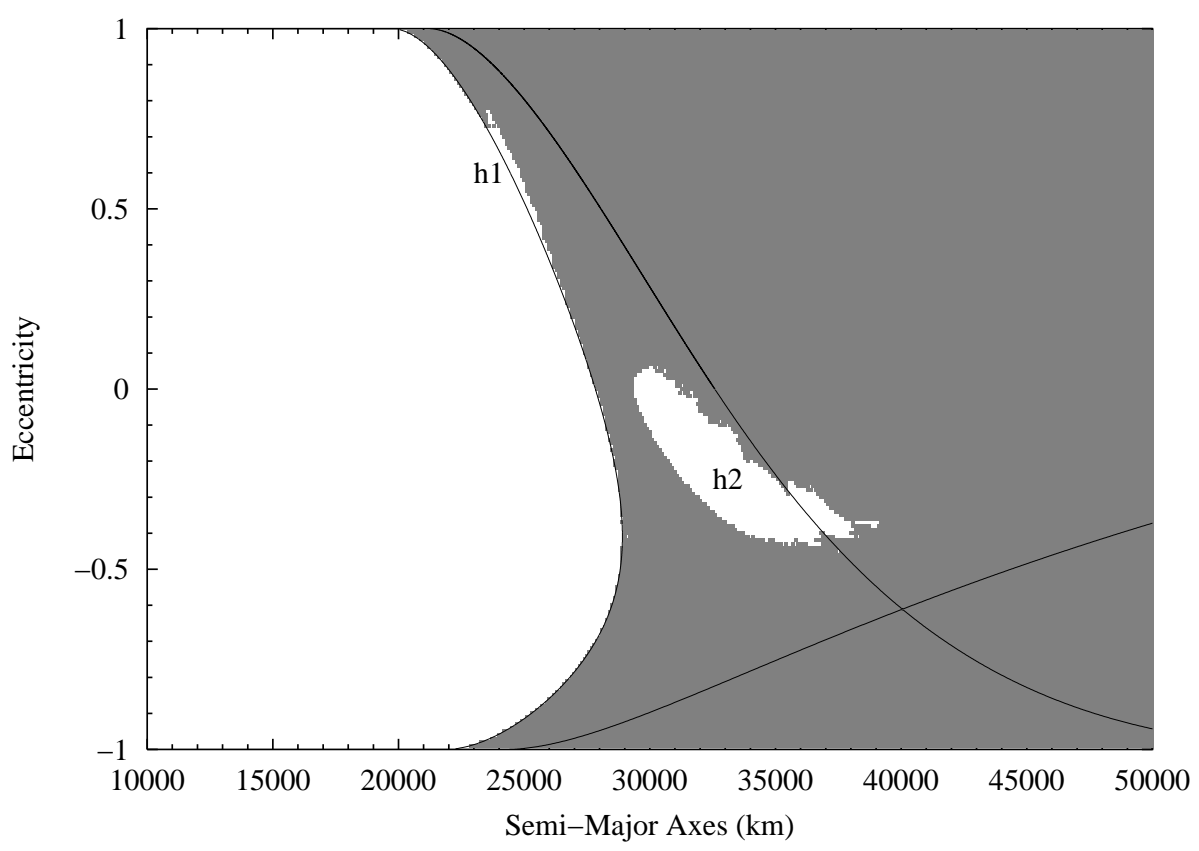

Fig. 2. Diagram of the initial conditions in terms of $a$ versus $e$. The black lines in the graphics stand for the zero velocity curves with $C j=C j(L 1)$ (most left line) and with $C j=C j(L 2)$ (two lines inside the gray region). The top half of this diagram $(e>0)$ corresponds to conditions at pericenter at opposition ( $\omega=0$ and $\tau=0$, where $\tau$ is the pericenter's epoch), while the other half (e<0, this sign is just symbolic) corresponds to conditions at apocenter at opposition ( $\omega=\pi$ and $\tau=P / 2$, where $P$ is the osculating orbital period). Initial conditions whose trajectories presented capture times longer than $10^{4}$ days are indicated by the white areas (inside the gray region) labeled $h 1$ and $h 2$.
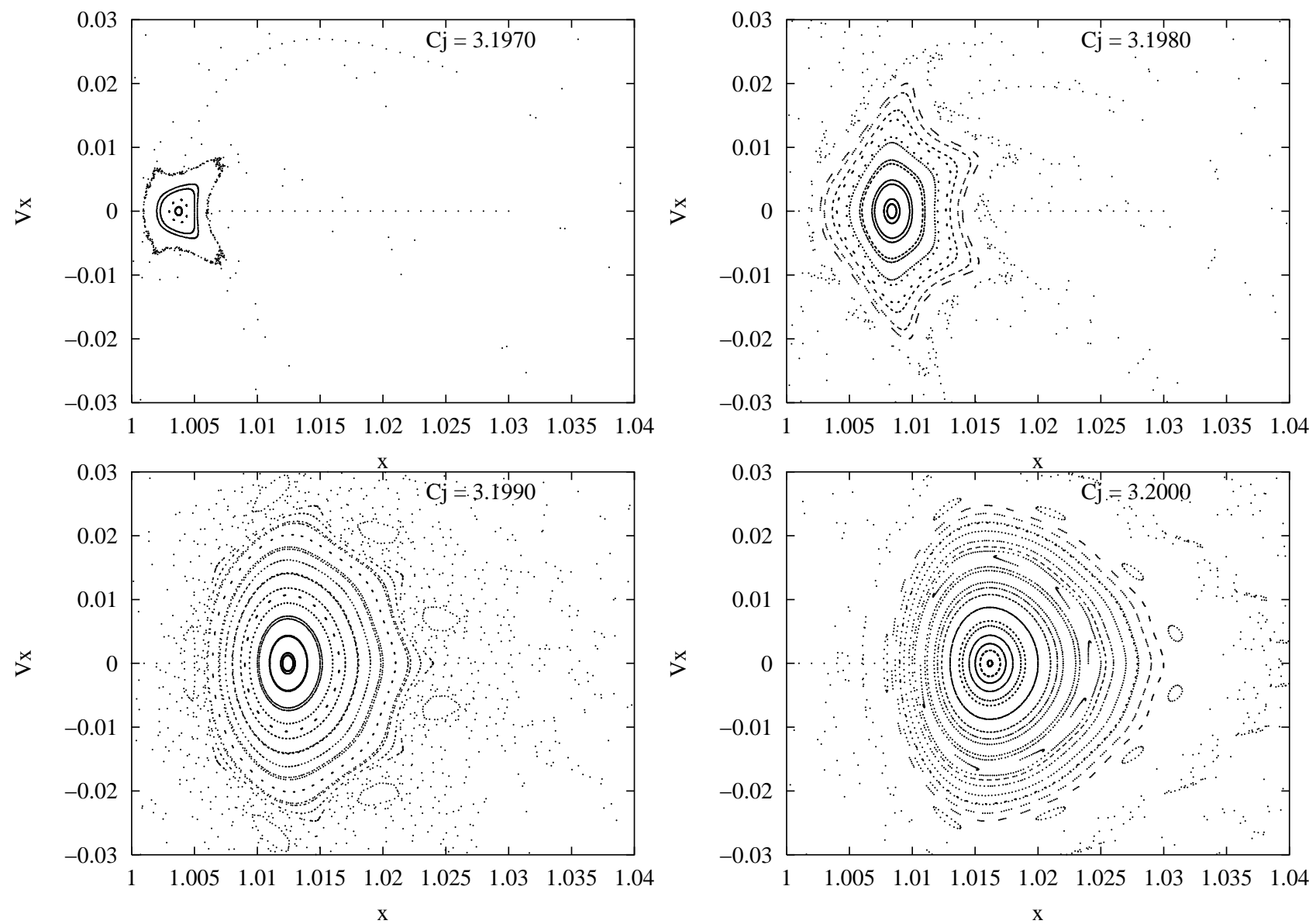

Fig. 3. Set of Poincaré surfaces of section for different values of Jacobi constant, $3.197 \leq C_{j} \leq 3.200$. The range of $C_{j}$ considered in these plots coincides with the range needed to cover the area $h 1$ given in Fig. 2. The center of the concentric islands shown in each surface of section corresponds to one periodic orbit of the family $h 1$ (Broucke 1968). 

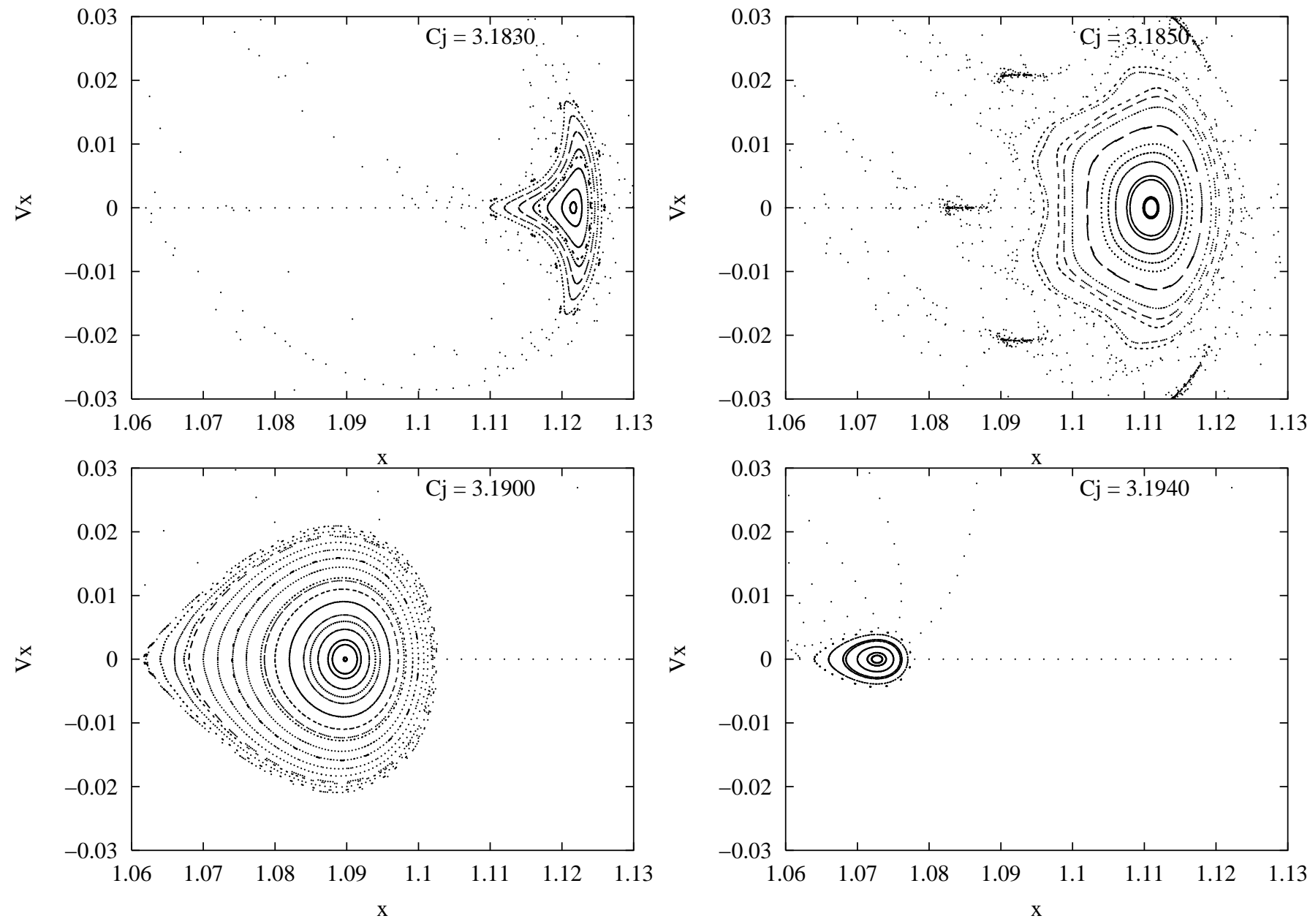

Fig. 4. Set of Poincaré surfaces of section for different values of Jacobi constant, $3.183 \leq C_{j} \leq 3.194$. The range of $C_{j}$ considered in these plots coincide with the range needed to cover the area $h 2$ given in Fig. 2. The center of the concentric islands shown in each surface of section corresponds to one periodic orbit of the family $h 2$ (Broucke 1968).

stable regions. These stable regions are associated with periodic orbits.

\subsection{Periodic orbits}

The center of the islands for each surface of section corresponds to one periodic orbit. They are members of two families of simple direct periodic orbits called $h 1$ and $h 2$ (Broucke 1968), also called families $g 1$ and $g 2$ (Hénon 1970) or $S$ and $N$ (Gorkavyi 1993). They were initially classified in a single family, called family $g$ (Broucke 1962; Szebehely 1967). It is important to note that in these previous works the main goal was to study the periodic orbits. None of them paid much attention to the quasi-periodic orbits, their size and location.

A sample of trajectories of the family of periodic orbits $h 1$ is given in Fig. 5. The trajectories chosen are four periodic orbits that appeared in Fig. $3\left(C_{j}=3.197, C_{j}=3.198\right.$, $C_{j}=3.199$ and $C_{j}=3.200$ from left to right). The thick black lines correspond to the zero velocity curves that touch the points $L_{1}$ or $L_{2}$. In Fig. 6 are presented trajectories of the four periodic orbits of family $h 2$, that appeared in Fig. 4
$\left(C_{j}=3.183, C_{j}=3.185, C_{j}=3.190\right.$ and $C_{j}=3.194$ from right to left). A comparison between the two families shows that the periodic orbits of family $h 1$ have their closest approach to the Moon at opposition while for orbits of family $h 2$ it happens at inferior conjunction. Another feature is that the periodic orbits of family $h 1$ are more elongated than those of family $h 2$.

\subsection{Quasi-periodic orbits}

The islands in the Poincaré surface of section correspond to quasi-periodic orbits around the periodic one located in the center of the respective islands. The largest of these islands are those with the maximum amplitude of oscillation that are still stable. In order to visualize the shape and evolution of such orbits we selected two of them. In Fig. 7 we present the trajectory of one quasi-periodic orbit around a periodic orbit of family $h 1$ that appeared in Fig. 4 (largest island at $C_{j}=3.200$ ). In Fig. 8 we present the trajectory of one quasi-periodic orbit around a periodic orbit of family $h 2$ that appeared in Fig. 5 (largest island at $C_{j}=3.190$ ). Despite of the fact that these trajectories have Jacobi constant values enough to escape. In both cases 


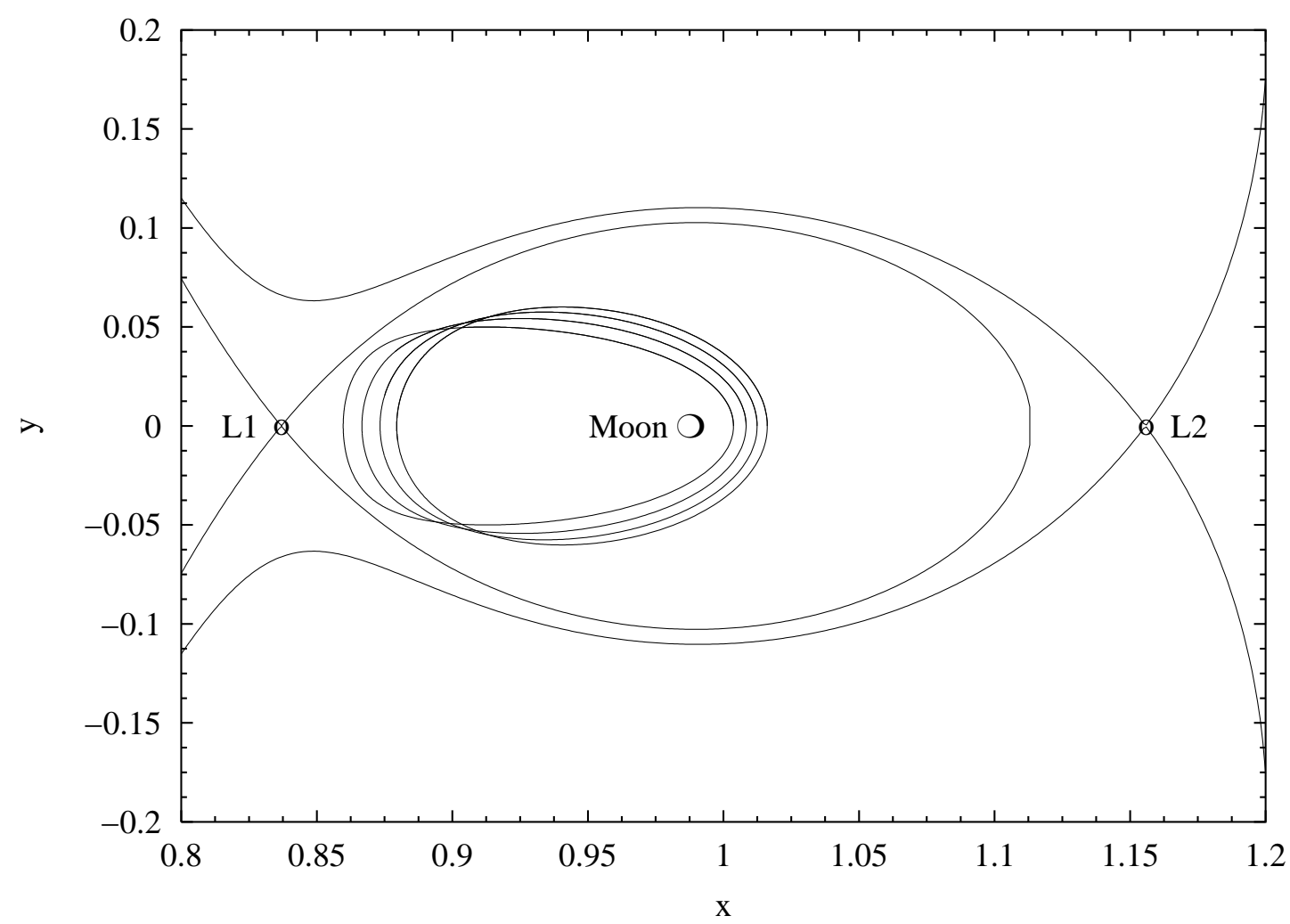

Fig. 5. Trajectories of the four periodic orbits of family $h 1$, that appeared in Fig. $3\left(C_{j}=3.197, C_{j}=3.198, C_{j}=3.199\right.$ and $C_{j}=3.200$ from left to right).

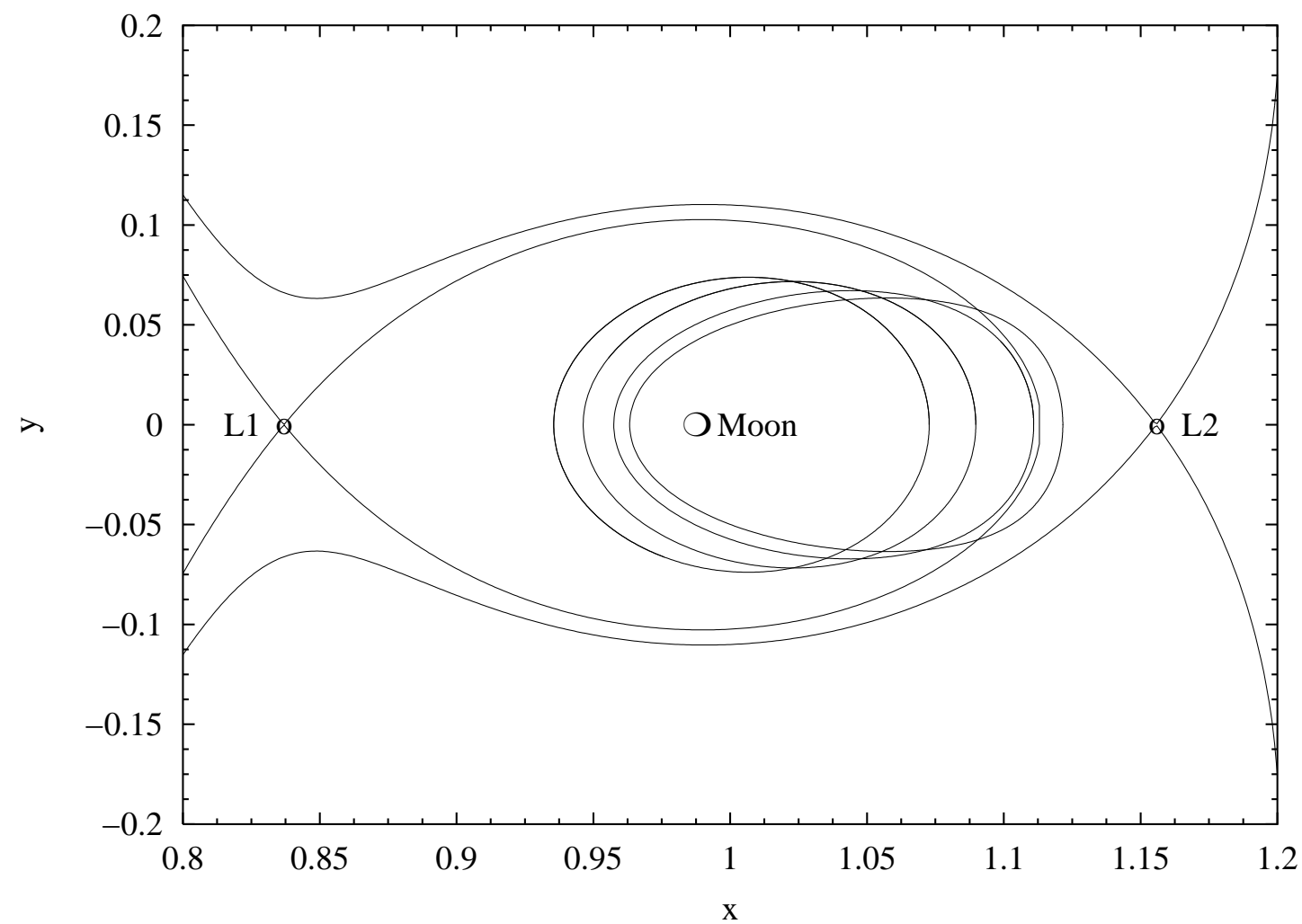

Fig. 6. Trajectories of the four periodic orbits of family $h 2$, that appeared in Fig. $4\left(C_{j}=3.183, C_{j}=3.185, C_{j}=3.190\right.$ and $C_{j}=3.194$ from right to left). 


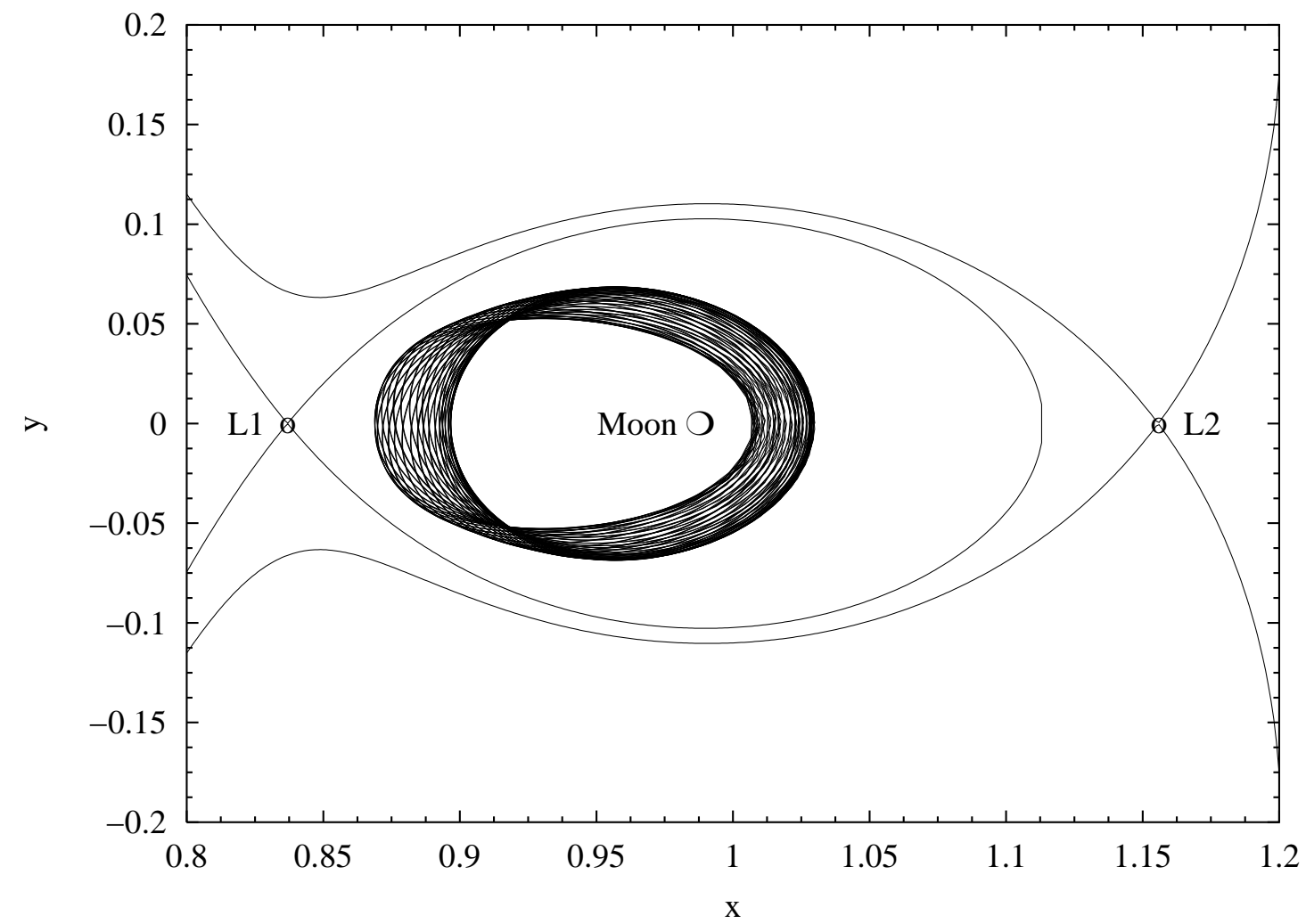

Fig. 7. Trajectory of one quasi-periodic orbit around a periodic orbit of family $h 1$ that appeared in Fig. 4 (largest island at $C_{j}=3.200$ ).

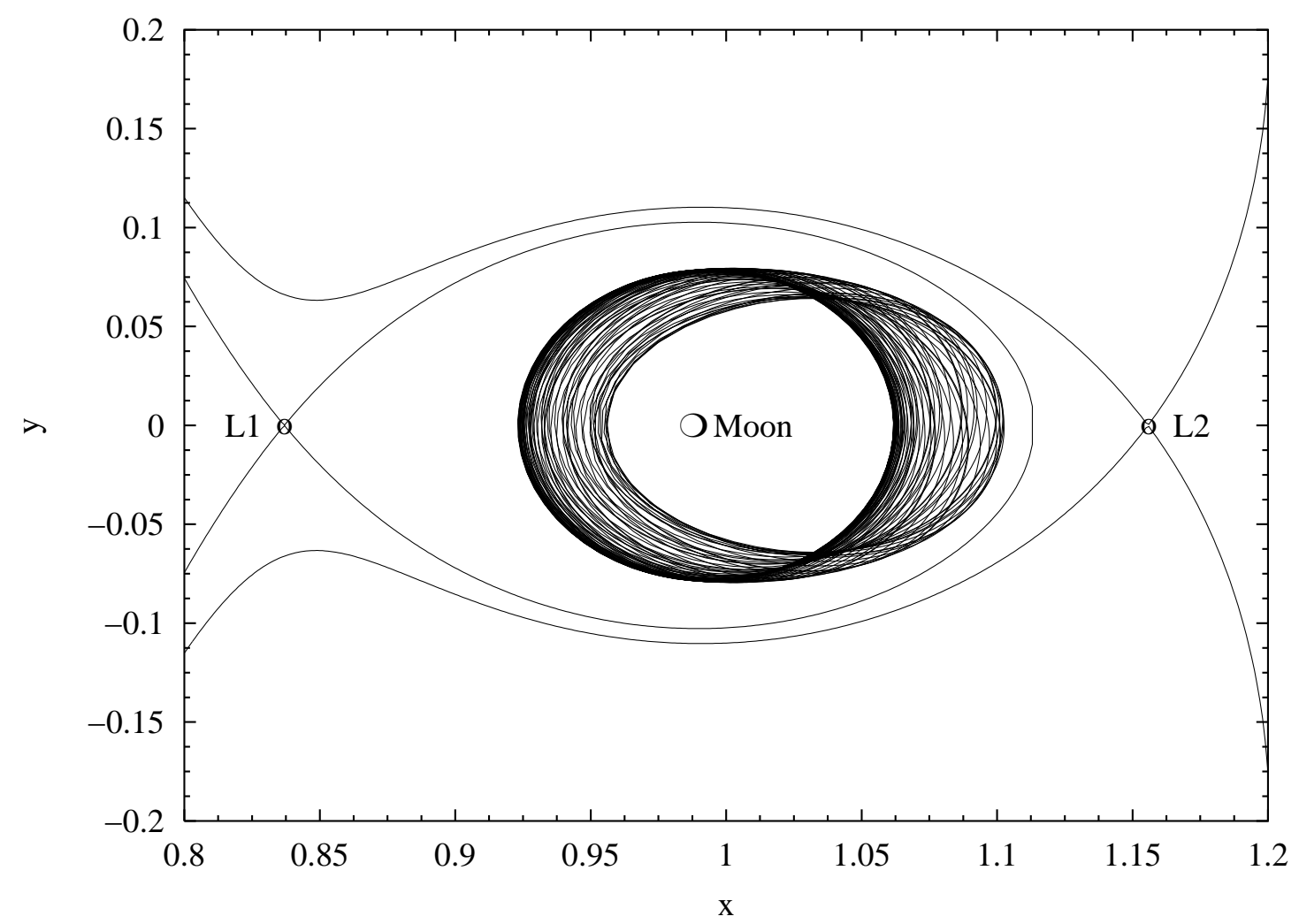

Fig. 8. Trajectory of one quasi-periodic orbit around a periodic orbit of family $h 2$ that appeared in Fig. 5 (largest island at $C_{j}=3.190$ ). 


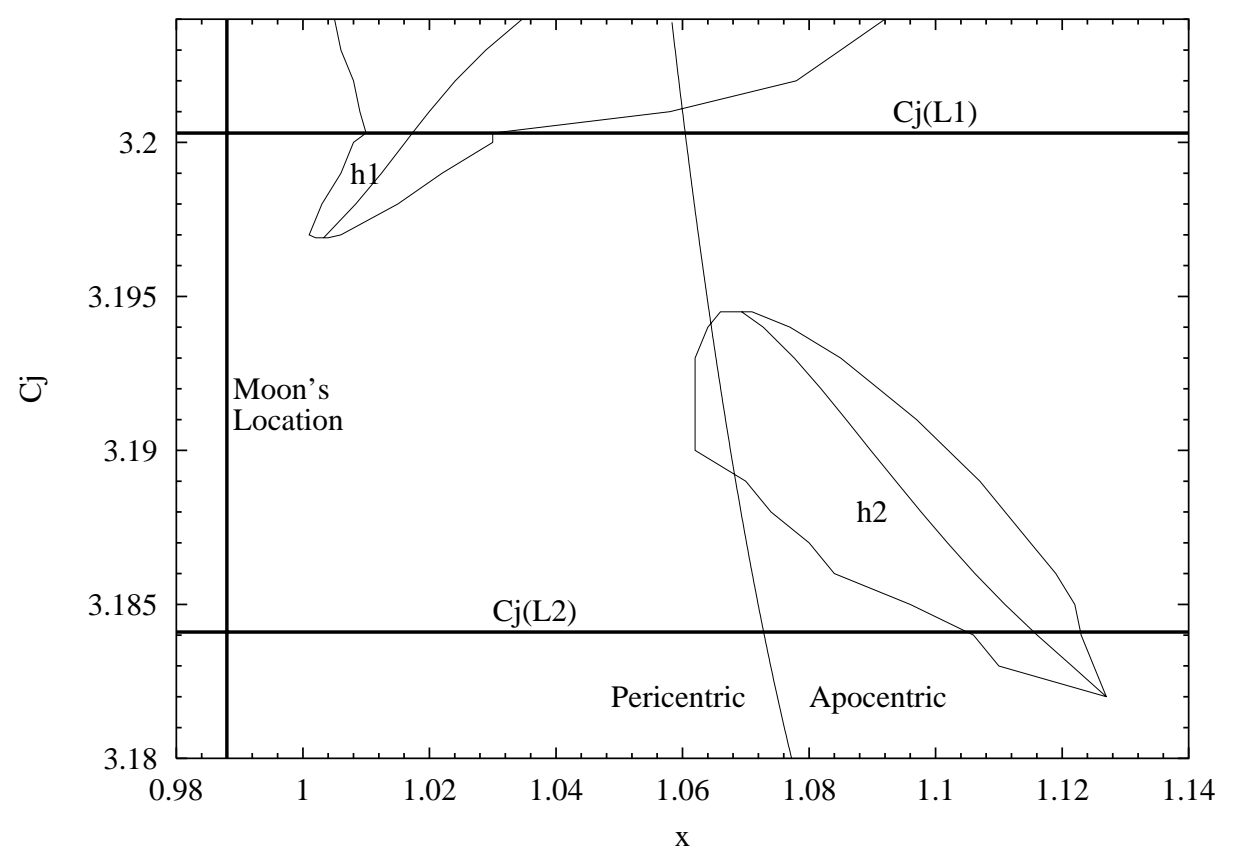

Fig. 9. Stability diagram showing the location and width of the maximum amplitude of oscillation around the periodic orbit $h 1$ and $h 2$ as a function of the Jacobi constant. The three thick black lines indicate the Moon's location and the values of the Jacobi constant at points $L_{1}$ or $L_{2}$. The thin line that crosses the figure corresponds to circular $(e=0)$ trajectories, and divide the figure into pericentric (left) and apocentric (right) initial conditions.

the trajectories remain inside the Roche lobe and also maintain the shape of the periodic orbit associated with each one. In astrodynamics applications this kind of orbital characteristic is valuable. These stable regions could be used to keep missions around the Moon, at a far distance, based on a very low fuel consumption.

\section{Stability criterion}

In the present dynamical system the largest of the quasiperiodic orbits corresponds to the maximum amplitude of oscillation around the periodic one. Following the approach adopted by Winter (2000), the stability of these kinds of periodic orbits can be measured in terms of the maximum amplitude of oscillation about the periodic orbit. This maximum amplitude of oscillation can be quantified from the Poincaré surface of section considering, for each Jacobi constant, the width of the largest island (quasi-periodic orbit) in the line of conjunction (values of $x$ when $\dot{x}=0)$.

We have measured such amplitudes from our surfaces of section and the results are presented in Fig. 9. The thick lines indicate the location of the Moon and the values of the Jacobi constant associated with the Lagrangian points $L 1$ and $L 2$. There is a thin line that divides the figure into pericentric and apocentric regions. The thin lines labeled $h 1$ and $h 2$ indicate the location of the periodic orbits, while the lines around them indicate the maximum amplitude of oscillation in each case. From this figure we can see the evolution of the stability for both families of periodic orbits as a function of the Jacobi constant. In the case of the periodic orbit $h 1$ there is no stability region (in the sense defined by Winter 2000) for $C_{j} \leq 3.1969$.
In the case of $h 2$, the stability region is confined to the interval $3.1820 \leq C_{j} \leq 3.1945$.

\section{Robustness of the stability}

The results obtained in the previous sections are in the framework of the restricted three-body problem, Earth-MoonParticle. However, the real system involves some perturbations that could affect the stability of the regions studied. One kind of perturbation that could be relevant is the tidal force produced by the Moon on the Earth. As a result of such force, the orbit of the Moon evolves outward. However, the effects due to the tidal force become relevant only on relatively large time scales. For instance, the rate at which the Moon moves away from the Earth is about $4 \mathrm{~cm}$ per year. Therefore, it will take about $2.5 \times 10^{4}$ years for the orbit of the Moon to move just one kilometer. Since we are dealing with stability on a time scale of the order of a few years, it is reasonable to neglect the tidal effects. On the other hand, a perturbation that can actually affect the stability regions is the solar gravitational field.

\subsection{Solar perturbations}

In order to investigate the effects of solar perturbations we numerically simulated and compared two dynamical systems: the restricted three-body problem, Earth-Moon-Particle, and the restricted four-body problem, Sun-Earth-Moon-Particle. The range of initial conditions considered covers the area of the region $h 2$. In fact the stable region $h 2$ is the one that might be more useful for practical purposes, since it is much larger than the region $h 1$. Different from the results presented in Fig. 2, we 

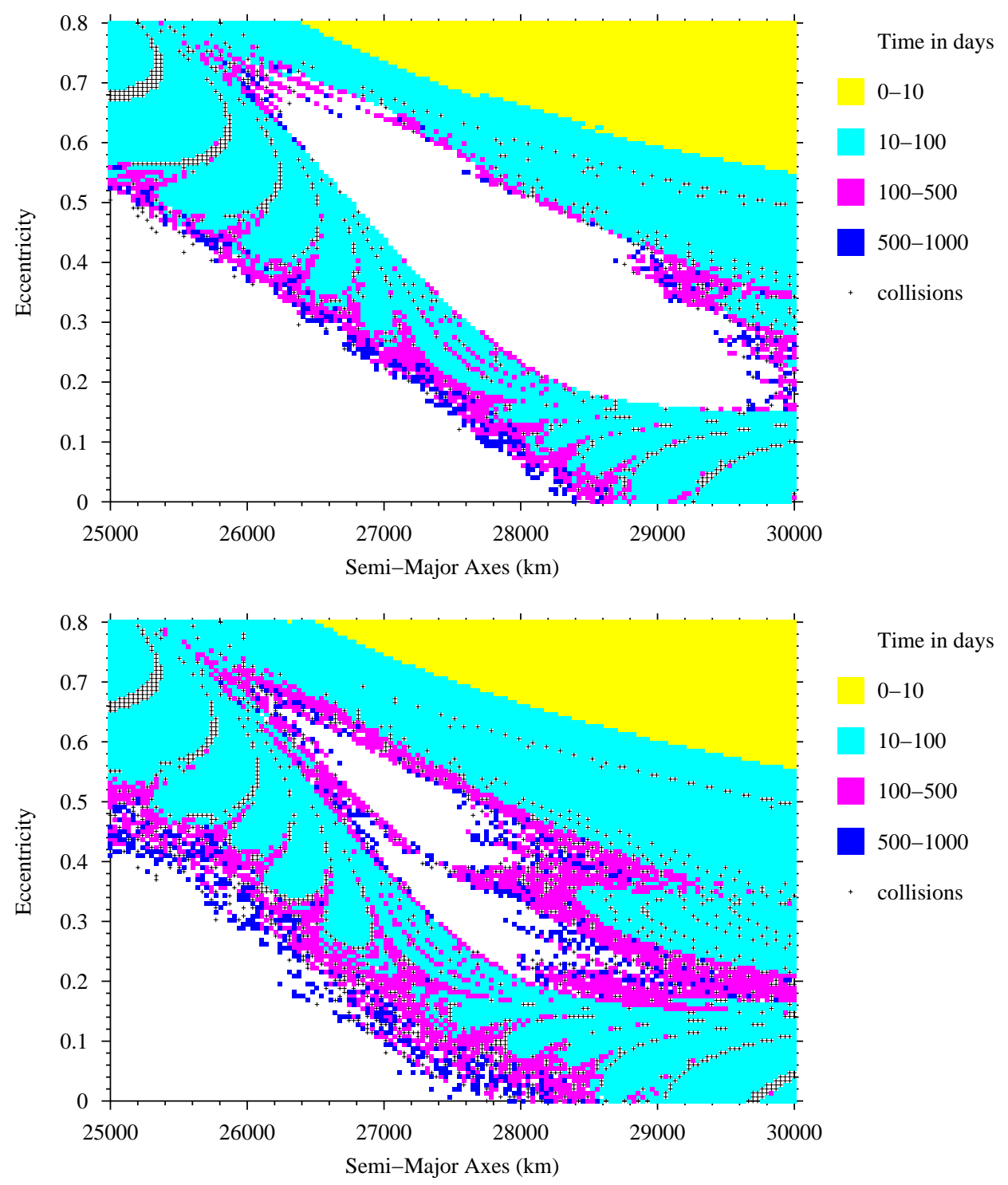

Fig. 10. Stability region in terms of capture times. Each initial condition has its capture time indicated by the gray coded color. The white areas inside the gray region correspond to initial conditions that remain captured for the whole period of integration ( $10^{3}$ days). Two dynamical systems were considered: a) Restricted three-body problem, Earth-Moon-particle (top). b) Restricted four-body problem, Sun-Earth-Moonparticle (bottom).

considered the initial conditions at pericenter at inferior conjunction ( $\omega=\pi$ and $\tau=0$ ) in order to be able to see the whole stable region of $h 2$ at pericenter. The time span was reduced to $10^{3}$ days since that is the length of time useful for practical purposes. In Fig. 10 we present the results in the initial conditions frame $a \times e$ in terms of capture times. The top figure corresponds to the results on the Earth-Moon-Particle system while in the bottom figure the solar perturbation is included. A comparison of such figures clearly show that the solar perturbation is not negligible. It reduces the size of the stable region, whose trajectories remain captured for more than a thousand days (white area inside the gray region). Actually, it almost divides that region into two parts. Nevertheless, the size of the stable region is still significant. The solar perturbation reduces the size of the stable region to about half of its size.
The effects of gravitational attraction of the Sun on the trajectories of the stable region can be noted in two examples presented in Figs. 11 and 12. First, it is important to note that, as we have shown in the previous sections, the trajectories of the stable region found in the Earth-Moon-particle system are periodic/quasi-periodic orbits. Therefore, those trajectories will never escape, and the region is "stable for ever". On the other hand, the trajectories of the stable region found in the Sun-Earth-Moon-particle system are not periodic/quasiperiodic orbits. Those are trajectories that remain captured for the whole time of integration $\left(10^{3}\right.$ days). So, they are stable in the sense that they do not escape. In order to see the typical behaviour of such trajectories we chose two initial conditions that are stable in both systems: 1) $a=27,751.7 \mathrm{~km}$ and $e=0.3227$ (Fig. 11). 2) $a=27,248.3 \mathrm{~km}$ and $e=0.4638$ (Fig. 12). In 

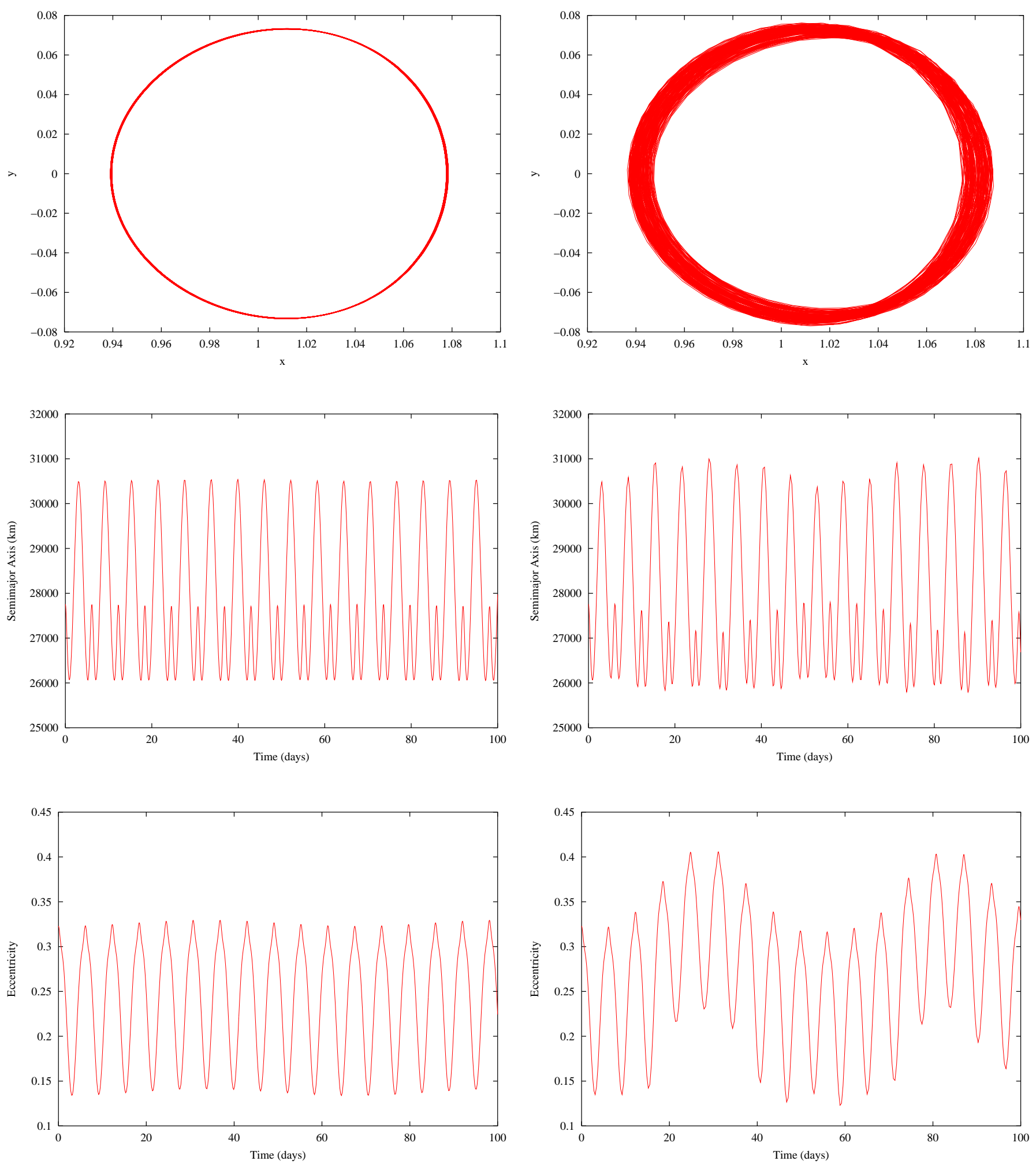

Fig. 11. Example of a trajectory with initial conditions $a=27,751.7 \mathrm{~km}$ and $e=0.3227$, which is stable in both systems: Earth-Moon-particle (left column), and Sun-Earth-Moon-particle (right column). In the first row is given the trajectory in the baricentric rotating Earth-Moon system for one thousand days. The plots of the semimajor axis and the eccentricity are given for a time span of one hundred days, but the behaviour is the same for one thousand days.

the first case, Fig. 11, it can be seen that the solar perturbation increased the amplitude of oscillation and the complexity of the evolution of the semimajor axis and eccentricity. The trajectory in the baricentric rotating Earth-Moon system shows 

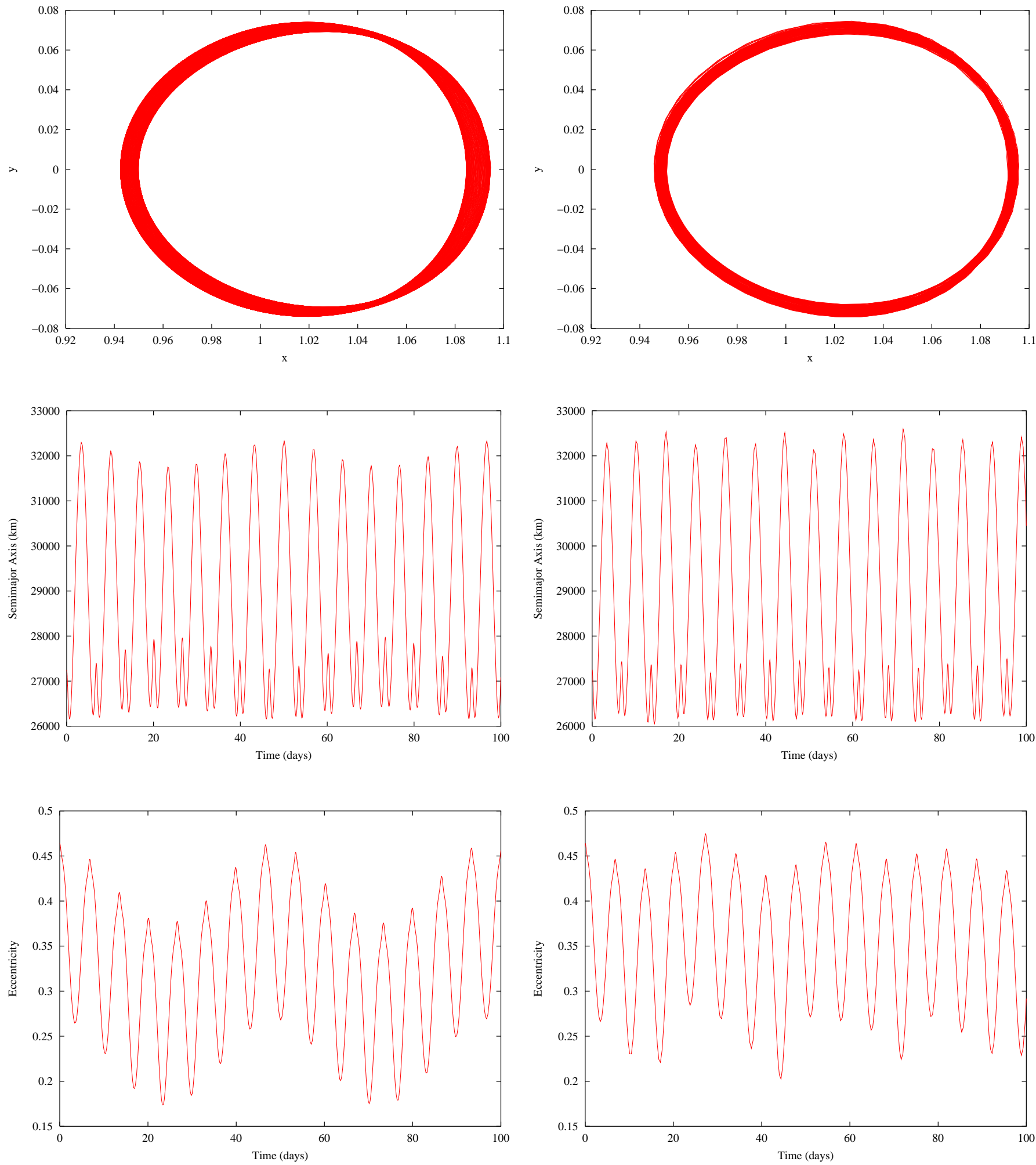

Fig. 12. Example of a trajectory with initial conditions $a=27,248.3 \mathrm{~km}$ and $e=0.4638$, which is stable in both systems: Earth-Moon-particle (left column), and Sun-Earth-Moon-particle (right column). In the first row is given the trajectory in the baricentric rotating Earth-Moon system for one thousand days. The plots of the semimajor axis and the eccentricity are given for a time span of one hundred days, but the behaviour is the same for one thousand days.

a significant increase in oscillation, but it maintains a regular the solar perturbation increased the complexity of the evolubehaviour for one thousand days. In the second case, Fig. 12, tion of the semimajor axis and eccentricity, but the amplitudes 
of oscillation are very close. The trajectory in the baricentric rotating Earth-Moon system shows a regular behaviour for one thousand days and, differently from the first case, its amplitude of oscillation decreased.

Therefore, including the solar perturbations there still exists a significant region where the trajectories show regular behaviour for at least a few years. This information can be useful in spacecraft missions. These "stable regions" could be used to keep missions around the Moon, at a far distance, based on a very low fuel consumption, for at least $10^{3}$ days.

Acknowledgements. This work was funded by Fapesp (Proc. 98/ 15025-7) and CNPq (Proc. 300347/01-4). These supports are gratefully acknowledged.

\section{References}

Broucke, R. 1962, Recherches d'orbites périodiques dans le problème restreint plan, Dissertation, Univ. of Louvain, Belgium

Broucke, R. 1968, JPL Techn. Rep., 32-1168

Brunini, A. 1996, Cel. Mec. Dyn. Astron., 64, 79

Gorkavyi, N. 1993, Astron. Lett., 19(6), 448

Hénon, M. 1970, A\&A, 9, 24

Huang, T. Y., \& Innanen, K. A. 1983, AJ, 88 (10)

Jefferys, W. H. 1971, An Atlas of Surfaces of Section for the Restricted Three Bodies (University of Texas, Austin)

Roy, A. E., \& Ovenden, M. W. 1955, MNRAS, 115, 296

Szebehely, V. 1967, Theory of Orbits (Academic Press, New York)

Vieira Neto, E., \& Winter, O. C. 2001, AJ, 122(1), 440

Winter, O. C. 2000, Planet. Sp. Sci., 48, 23

Winter, O. C., \& Vieira Neto, E. 2001, A\&A, 377, 1119 